\title{
Surface Display of Recombinant Drosophila melanogaster Acetylcholinesterase for Detection of Organic Phosphorus and Carbamate Pesticides
}

\author{
Jingquan $\mathrm{Li}^{1,39}$, Qian $\mathrm{Ba}^{1,39}$, Jun $\mathrm{Yin}^{19}$, Songjie $\mathrm{Wu}^{1}$, Fangfang Zhuan ${ }^{1}$, Songci $\mathrm{Xu}^{1}$, Junyang $\mathrm{Li}^{1}$, \\ Joelle K. Salazar ${ }^{2}$, Wei Zhang', Hui Wang ${ }^{1,3 *}$
}

1 Key Laboratory of Food Safety Research, Institute for Nutritional Sciences, Shanghai Institutes for Biological Sciences, Chinese Academy of Sciences, Shanghai, P. R. China, 2 Institute for Food Safety and Health, Illinois Institute of Technology, Bedford Park, Illinois, United States of America, 3 Key Laboratory of Food Safety Risk Assessment, Ministry of Health, Beijing, P. R. China

\begin{abstract}
Acetylcholinesterase (AChE) is commonly used for the detection of organophosphate (OP) and carbamate (CB) insecticides. However, the cost of this commercially available enzyme is high, making high-throughput insecticide detection improbable. In this study we constructed a new AChE yeast expression system in Saccharomyces cerevisiae for the expression of a highly reactive recombinant $\mathrm{AChE}$ originating from Drosophila melanogaster (DmAChE). Specifically, the coding sequence of DmAChE was fused with the 3 '-terminal half of an $\alpha$-agglutinin anchor region, along with an antigen tag for the detection of the recombinant protein. The target sequence was cloned into the yeast expression vector pYes-DEST52, and the signal peptide sequence was replaced with a glucoamylase secretion region for induced expression. The resultant engineered vector was transformed into S. cerevisiae. DmAChE was expressed and displayed on the cell surface after galactose induction. Our results showed that the recombinant protein displayed activity comparable to the commercial enzyme. We also detected different types of OP and CB insecticides through enzyme inhibition assays, with the expressed DmAChE showing high sensitivity. These results show the construction of a new yeast expression system for DmAChE, which can subsequently be used for detecting $\mathrm{OP}$ and $\mathrm{CB}$ insecticides with reduced economic costs.
\end{abstract}

Citation: Li J, Ba Q, Yin J, Wu S, Zhuan F, et al. (2013) Surface Display of Recombinant Drosophila melanogaster Acetylcholinesterase for Detection of Organic Phosphorus and Carbamate Pesticides. PLoS ONE 8(9): e72986. doi:10.1371/journal.pone.0072986

Editor: Israel Silman, Weizmann Institute of Science, Israel

Received February 13, 2013; Accepted July 23, 2013; Published September 9, 2013

Copyright: (c) $2013 \mathrm{Li}$ et al. This is an open-access article distributed under the terms of the Creative Commons Attribution License, which permits unrestricted use, distribution, and reproduction in any medium, provided the original author and source are credited.

Funding: This study was supported by grants from One Hundred Talents Program of the Chinese Academy of Sciences, the Science and Technology Commission of Shanghai Municipality (10391902100, 08391910800), the National Nature Science Foundation $(81125020,91029715,31070680$ and 31101261), the Ministry of Science and Technology of China (2012BAK01B00, 2011BAK10B00 and 2009CB919000), the Science and Technology Commission of Xuhui District of Shanghai Municipality (RCT201001), and Xuhui Central Hospital (CRC2010002, CRC2011001 and CRC2011004), Director Foundation (20090101) and the Food Safety Research Center and Key Laboratory of Nutrition and Metabolism of INS, SIBS, CAS. The funders had no role in study design, data collection and analysis, decision to publish, or preparation of the manuscript.

Competing Interests: The authors have declared that no competing interests exist.

*E-mail: huiwang@sibs.ac.cn

9 These authors contributed equally to this work.

\section{Introduction}

Organophosphate $(\mathrm{OP})$ and carbamate $(\mathrm{CB})$ insecticides have been widely used in the agricultural industry to increase the yield of crops $[1,2]$. These pesticides are applied to plant surfaces or the soil itself to kill target organisms, mainly insects. However, OP and $\mathrm{CB}$ insecticides have been shown to be harmful on the environment, agricultural workers, and the surrounding human and animal population [2]. It is known that the toxicity of OP and CB insecticides is mainly due to the inhibition of a key enzyme, acetylcholinesterase (AChE, EC3.1.1.7), that plays a role in neural conduction pathways [3-5]. AChE hydrolyzes the important neurotransmitter acetylcholine, which assures the normal conduction of nerve impulses [6-8]. OP and CB insecticides bind to AChE and inhibit the native hydrolytic ability through phosphorylating the serine residues in the hydroxyl group [8]. After AChE is inhibited, acetylcholine accumulates in the synapse and results in blocked neural conduction pathways [9-14].
Many detection methods are currently available as a measure of protection against the harmful effects of $\mathrm{OP}$ and $\mathrm{OB}$ insecticides. However, a novel system that would detect residual OP and CB insecticides quickly and accurately remains to be developed. The more routine detection methods are commonly based on spectrum and chromatographic techniques [15-18], which are costly and time-consuming due to complex experimental procedures. Recently, AChE-based analytical methods have gained increasing interest due to the high speed and sensitivity attributed to on-site detection. According to previous studies, insect-originated AChE is more sensitive and more suitable to be used for detection of $\mathrm{OP}$ and $\mathrm{CB}$ insecticides compared with mammal-originated AChE [19]. However, the experimental procedures for extraction and purification of AChE from insects are time consuming and costly. To overcome these problems, different expression systems have been used, such as vaculovirus, yeast, Xenopus, oocytes and mammalian cells [20-25]. Among them, the yeast expression systems have the advantage of high enzymatic yields and stable fermentation characteristics [22]. 

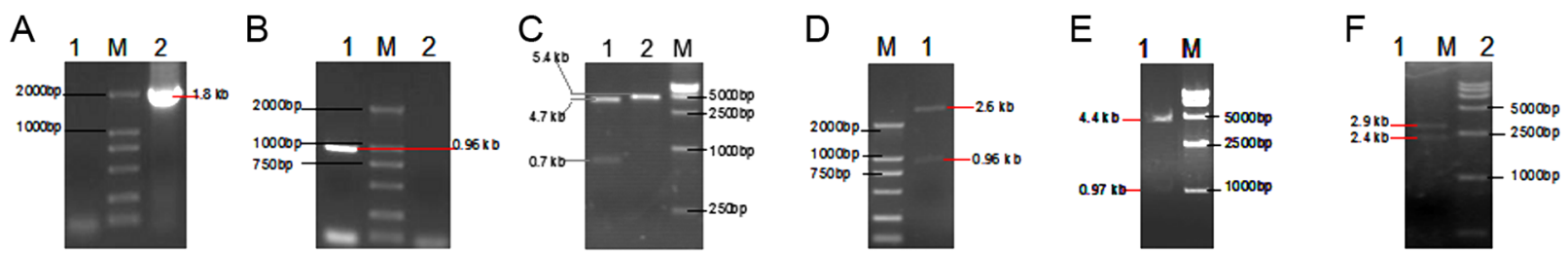
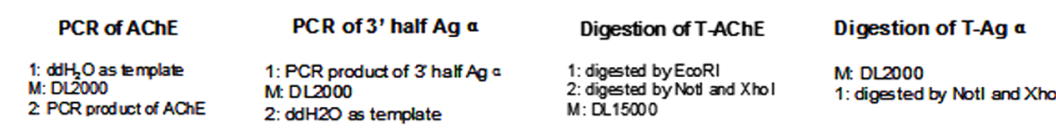

Digestion of TAChEflag-Ag a 1: digested by Notl and tho M. DL 15000

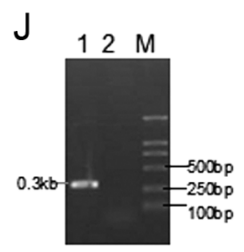

PCR of GSO1 1: digested by EooRV

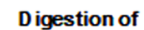
pYesAChEflagAga M. DL 15000

$\mathrm{H}$
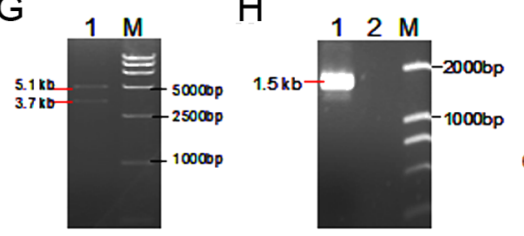

PCR of GSU

M: DL2000 1: PCR product of GSU by pGSU F1 and PGSU-R
2: ddH $O$ os template

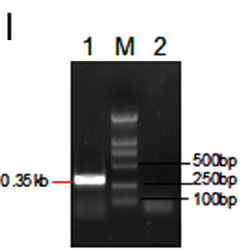

PCR of GSD2

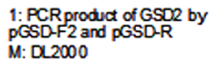
M: DL2000
2: $\mathrm{dH} 20$
$\mathrm{K}$

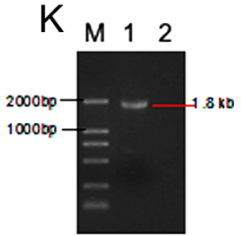

Overlap PCR of GS

1. overlap PCR product of GS 2: $\mathrm{ddH} 2 \mathrm{O}$ as template M:DL2000 1: PCR product of GSD1 by 2 ddH $2 \mathrm{O}$

M:DL2000 lomplato

\section{Digestion of}

1: digested by EcoRV M: DL15000

L

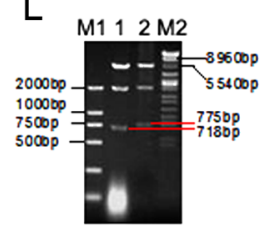

\author{
Digestion of psYes-AchEflag-Ag a \\ 1: psYES-AChE-Aga digested by Pvu II \\ and $\mathrm{Bgl}$ II \\ 2:pYES-DEST-AChE-Aga digested by PVu II \\ and BgI II \\ M1:DL2000 \\ M2:ADNA digested by Hind III,Bam HI,EcoRI
}

Figure 1. The identification of PCR constructs. doi:10.1371/journal.pone.0072986.g001

In the current yeast expression system, AChE is synthesized and secreted under certain conditions in a commercially available detection kit. AChE dry powder is commonly used due to its high stability and purity. However, the separation and purification of secreted enzyme is methodologically complex. Therefore, there is an urgent need to improve the existing yeast expression system to produce usable enzyme directly. The yeast surface display technique could very well be the appropriate solution. This technique has great potential in facilitating the production of sufficient usable quantities of functional AChE because yeast itself could be regarded as an immobilization carrier.

In the current study, we applied the surface display technique in the DmAChE yeast expression system and determined the production and activity of DmAChE. In our research, we fused the ace gene of $D$. melanogaster with a yeast $\alpha$-agglutinin gene to allow expression of DmAChE on the surface of yeast [26-29]. After inducement and fermentation, the yeast suspension can be used directly for $\mathrm{OP}$ and $\mathrm{CB}$ insecticides detection, with the activity and sensitivity comparable to the commercial enzyme at a significantly lower cost.

\section{Results}

\section{The identification of clones}

To ensure that the constructed vector contained the correct fragments, PCR analysis was used as verification during all construction steps. The fragments of DmAChE (1.8-kb) and the $3^{\prime}$ of $\operatorname{Ag} \alpha(0.9-\mathrm{kb})$ were obtained from $D$. melanogaster and $S$. cerevisiae respectively (Figure $1 \mathrm{~A}$ and $1 \mathrm{~B}$, respectively). After digestion with appropriate restriction enzymes, the $4.7-\mathrm{kb}$ and $0.7-\mathrm{kb}$ fragments showed that the DmAChE fragment was inserted into pMD18-T accurately (Figure 1C), while the 0.96-kb and 2.6-kb fragments (Figure 1D) showed that the fragment was inserted into the $3^{\prime}$ of $\mathrm{Ag} \alpha$. Figure $1 \mathrm{E}$ and $1 \mathrm{~F}$ show that the FLAG region, together with the AChE-Ag $\alpha$ fragments, were inserted into pMD18-T and pENTR $^{\mathrm{TM}}$ Directional TOPO. After recombination with pYes-
DEST52, the fragment containing DmAChE and the 3' of Ag $\alpha$, along with the FLAG, was inserted into pYes-DEST52 (Figure 1G). The 1.4-kb GSU (Figure 1H) and the 0.4-kb GSD2 (Figure 1I), which was amplified by PCR using GSD1 (Figure $1 \mathrm{~J}$ ) as template, were ligated by overlap PCR to get GS (Figure 1K). GS was digested and ligated into the vector to get the final construct (Figure 1L).

\section{Optimization of the induction conditions}

To explore the optimal induction conditions, we investigated the effects of induction time, galactose concentrations, and different culture mediums on induced DmAChE expression through an orthogonal test assay. Growth in YPD medium was generally higher than that in SC medium (Figure 2A and 2B). The expression level of DmAChE in YPD medium was also generally higher than that in SC minimal medium (Figure 2C and 2D). The expression level induced with $4 \%$ galactose was higher than with $2 \%$ and $1 \%$ galactose in both SC minimal medium and YPD medium. And the increasing tendency of expression became weak after $8 \mathrm{~h}$. Considering that inducting for $12 \mathrm{~h}$ or $16 \mathrm{~h}$ was more difficult and that the DmAChE activities were not significantly higher than that of $8 \mathrm{~h}$, we chose $4 \%$ galactose and $8 \mathrm{~h}$ induction time in YPD medium as the optimal induction condition.

\section{DmAChE inhibition studies}

To investigate the potential application of DmAChE in OP and $\mathrm{CB}$ insecticide detection, we determined the sensitivity of DmAChE to each of nine types of insecticides according to GBT5009.199-2003, which is the national standard (Rapid determination for organophosphate and carbamate pesticide residues in vegetables) issued by the Standardization Administration of China, using $S$. cerevisiae suspension as the pure enzyme. And we found that the activity of DmAChE expressed on the surface of yeast significantly decreased after incubations with different pesticides (Figure 3). Although the inhibition effects varied in different pesticide, the enzyme showed good sensitivity to 
A
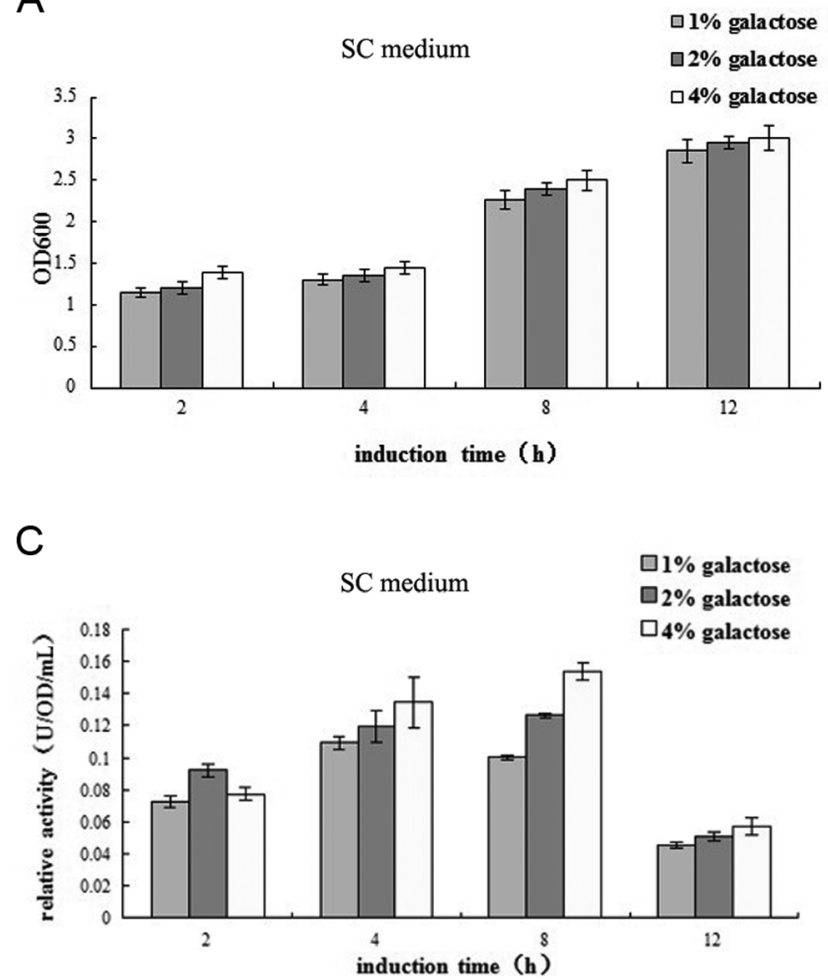

B

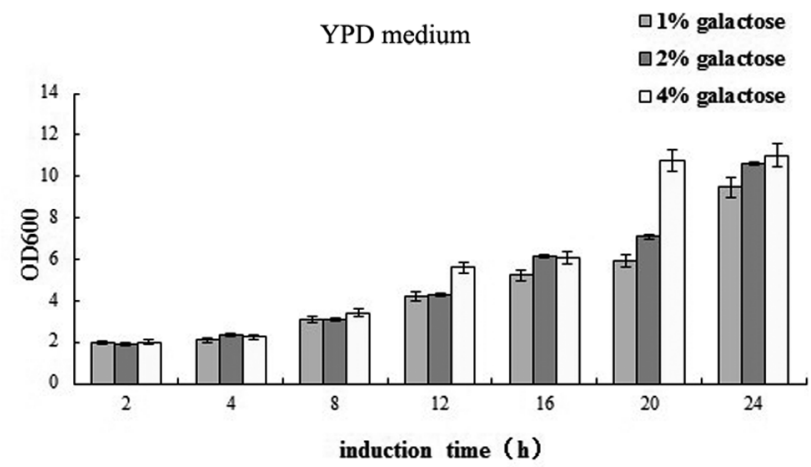

D
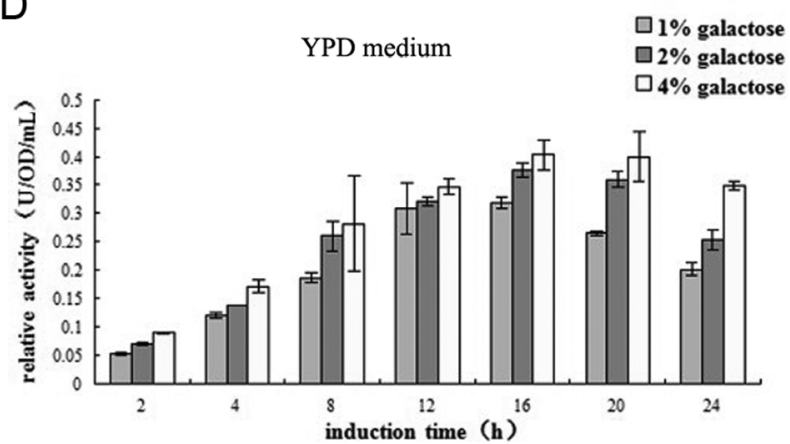

Figure 2. Analysis of the growth of yeast and the induced expression of AChE in different culture conditions. After induction in SC (A) and YPD (B) medium with $1 \%, 2 \%$, or $4 \%$ of galactose for different time periods, the $\mathrm{OD}_{600}$ values of yeast suspension were determined. After induction in SC (C) and YPD (D) minimal medium with 1\%, $2 \%$, or $4 \%$ of galactose for different time periods, the cultures were harvested and the relative enzyme activity of induced AChE was determined. Error bars represent the standard deviation of mean values. doi:10.1371/journal.pone.0072986.g002

most insecticides. Six pesticides could inhibit the activity more than $50 \%$ at $0.1 \mathrm{mg} / \mathrm{mL}$. Also, the inhibition effects of OP and $\mathrm{CB}$ insecticides showed in a concentration dependent manner (Figure 3). These results indicate that our expressed enzyme showed good sensitivity and can apply for the rapid determination for organophosphate and carbamate pesticide residues.

\section{Discussion}

In this study, we have described the expression of D. melanogaster AChE carrying the secretion and glycosylphosphatidylinositol (GPI)-anchoring signals in S. cerevisiae. Induced S. cerevisiae could serve as an expression system for functional AChE for the detection of $\mathrm{OP}$ and $\mathrm{CB}$ insecticides with relatively low detection limits. Thus, this study shows great potential for the further development of rapid detection technologies.

In the optimization of induction conditions, we speculated that the DmAChE activity was associated directly to expression level on the surface. We also speculated that the growth conditions of $S$. cerevisiae itself could influence the expression level directly. As in Figure 2A, S. cerevisiae enter into the logarithmic phase about $6 \mathrm{~h}$ after induction followed by stationary phase and death phase as a result of nutrient depletion in SC minimal medium. In comparison, the stationary phase and death phase is delayed in YPD medium (Figure 2A and 2B). DmAChE activity is directly proportional to the concentration of the inductor galactose. An exception to this was seen with $4 \%$ galactose and $2 \mathrm{~h}$ induction in $\mathrm{SC}$ medium. We speculated that this result is due to the short induction time, which greatly weakens the influence of high concentrations of inductor.
Recombinant DmAChE has been previously produced in Pichia pastoris $[28,30]$. In this study, the signal for the GPI anchor attachment was removed to induce DmAChE expression and secretion into the culture medium. However, the isolation and purification of recombinant DmAChE was costly. The yeast containing the surface enzyme could potentially be immobilized or used directly in insecticide detection. We compared our results with other reports using different enzymes. Firstly, our recombinant enzyme showed more sensitivity to carbaryl and carbofuran than natural AChE purified from different species including Oncorhynchus tshawytscha, Clarias batrachus, Electrophorus electricus and Bos Taurus [31,32]. Secondly, compared to recombinant enzymes such as Schizaphis graminum AChE and P. papatasi AChE produced in different baculovirus-based insect cells expression systems, our yeast-expressed AChE could be more effectively inhibited by paraoxon and/or carbaryl [33,34]. Even if the yeast-based recombinant $D$. melanogaster AChE expression system was also used, our surface display system showed more advantages and got better effects on monocrotophos and omethoate [30,35,36]. This might be due to the stability of the enzyme, in our system the yeast suspension was used directly, but not dried powder form in other studies. Our study provides for the possibility of the development of a biological sensor and other automatic detection techniques for a large number of agricultural products containing excess OP and CB insecticides. Through large scale fermentation, with omission of protein extraction and purification steps, the economic costs could decrease dramatically.

Compared to traditional chromatographic techniques, this system is more suitable for rapid detection strategies with excellent sensitivity. Other expression systems focus more on the yield and 

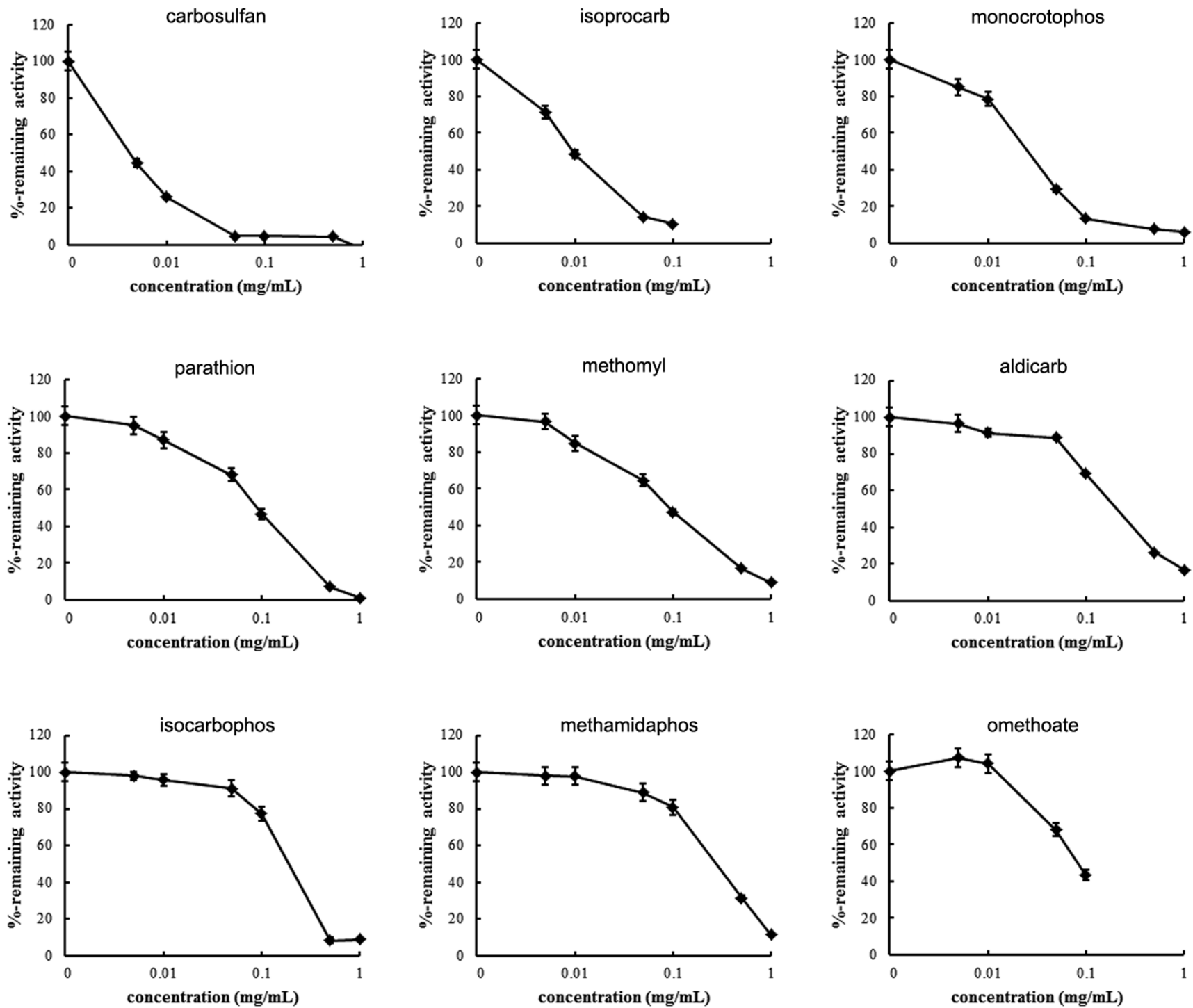

Figure 3. The inhibition curve of some organophosphorus and carbamate pesticides. The yeast with AChE expression were collected, resuspended in PBS buffer and seeded in 96-well plate. After incubation with various concentrations of different pesticides, the remaining enzyme activities were determined. Error bars represent the standard deviation of mean values.

doi:10.1371/journal.pone.0072986.g003

process of the recombinant enzyme [37-39] and less on the potential costs and time-consuming extraction and purification procedures after enzyme expression and secretion. There are also many studies regarding surface display systems, which we modified and applied to the current system of detection of OP and $\mathrm{CB}$ insecticides. As an immobilized carrier itself, yeast increases the stability of the enzyme and can be used directly as the functional enzyme in insecticide detection. Without the extraction and purification steps, the whole process from fermentation to acquisition takes $9 \mathrm{~h}$. Also, the activity of DmAChE in the yeast suspension could be maintained at more than 90\% following storage at $4{ }^{\circ} \mathrm{C}$ for 6 months (data not shown), which fits the requirement of fast detection methods.

In summary, the recombinant enzyme DmAChE was expressed and anchored on the surface of $S$. cerevisiae with the yeast suspension itself used for the detection of OP and CB insecticides directly with high stability. This system offers a promising alternative for the rapid detection of insecticidal residues.

\section{Materials and Methods}

\section{Strains and culture conditions}

$S$. cerevisiae INVSci of yeast was purchased from Invitrogen (Grand Island, NY). Cells were grown in Yeast Extract Peptone Dextrose (YPD) medium ( $1 \%$ yeast extract, $2 \%$ peptone, $2 \%$ dextrose) or Synthetic Complete (SC) minimal medium $(0.67 \%$ yeast nitrogen base, $2 \%$ carbon source, $0.01 \%$ of each adenine, arginine, cysteine, leucine, lysine, threonine, tryptophan, uracil, $0.005 \%$ of each aspartic acid, histidine, isoleucine, methionine, phenylalanine, proline, serine, tyrosine and valine). For selective media, uracil was omitted in SC. Competent Escherichia coli TOP10 and DH5 $\alpha$ were purchased from Tiangen (Beijing, China). Ampicillin, when necessary, was added to a final concentration of $100 \mu \mathrm{g} / \mathrm{mL}$. 
Total RNA from D. melanogaster
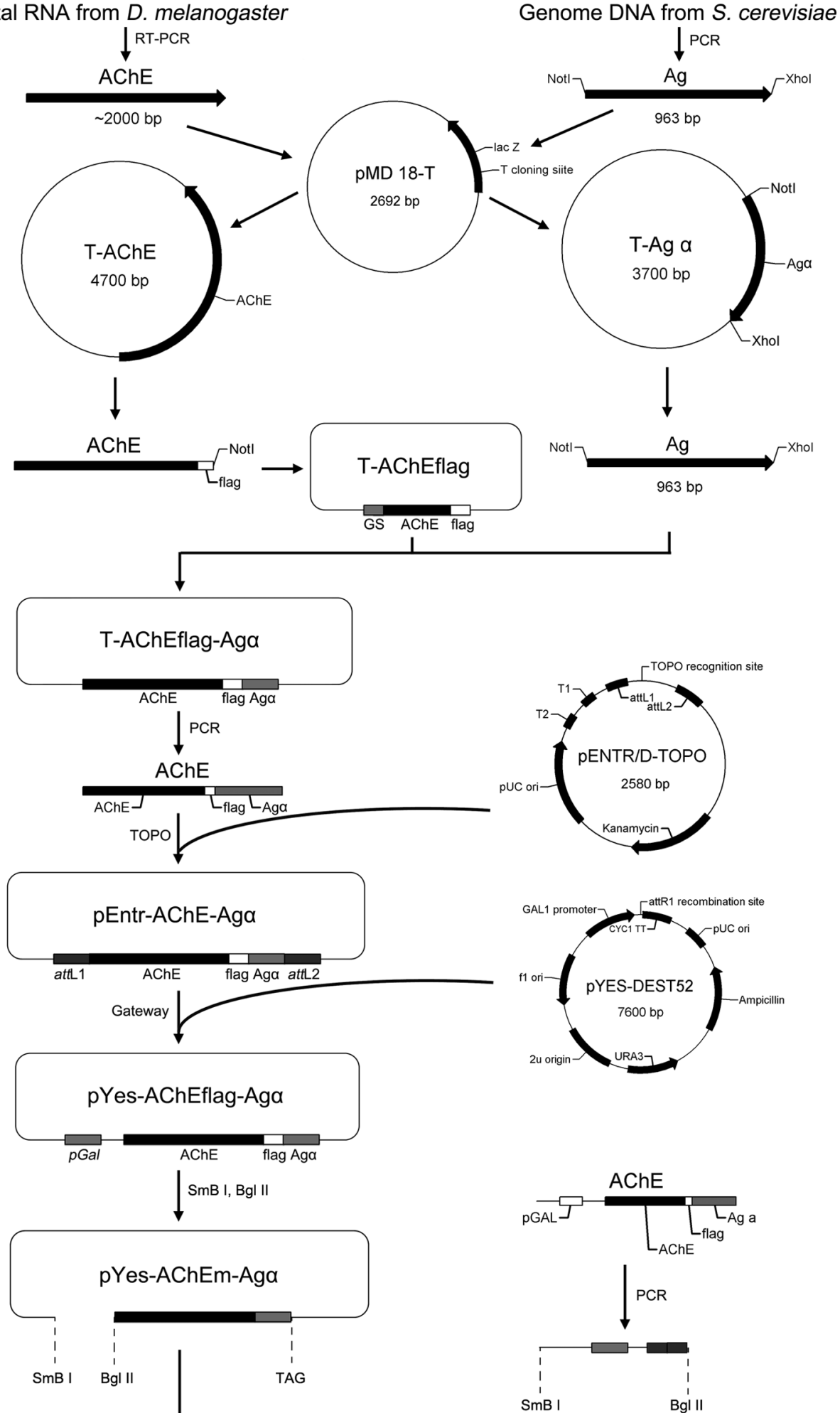
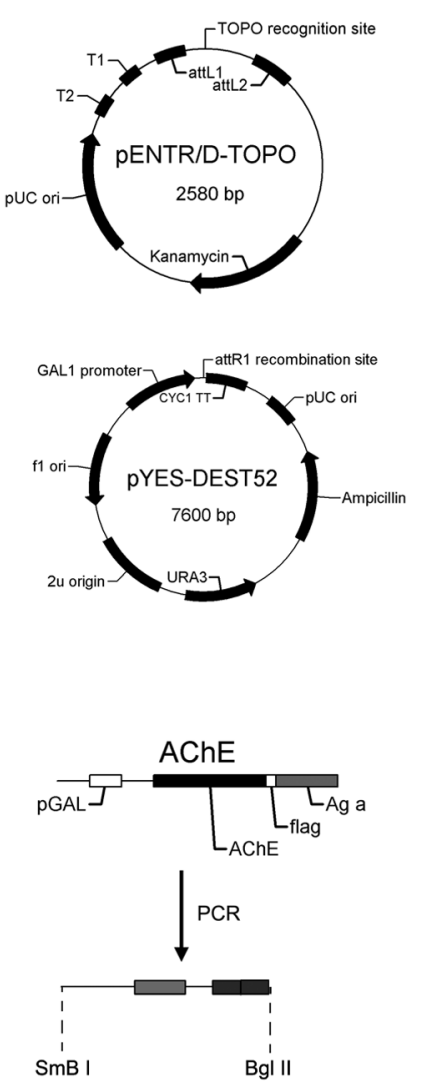
Figure 4. The construction of plasmid pYES-DEST52. doi:10.1371/journal.pone.0072986.g004

\section{Chemicals}

Acetylthiocholine iodide (ATChI), 5,5'-Dithiobis (2)-nitrobenzoic acid (DTNB), 1,5-Bis (4-allyldimethylammoniumphenyl) pentan-3-one dibromide (BW284c51) and tetraisopropyl pyrophosphoramide (iso-OMPA) were purchased from Sigma (St. Louis, $\mathrm{MO}$ ). $\mathrm{OP}$ and $\mathrm{CB}$ insecticides (purity $\geq 97.7 \%$ ) were purchased from Chinese Academy of Agricultural Sciences (Beijing, China).

\section{Plasmid construction}

D. melanogaster was kindly provided by Dr Jiankang Liu (Institute for Nutritional Sciences, Shanghai Institute of Biological Science, Chinese Academy of Sciences). DmAChE (Genbank ID: NM_057605) and the $3^{\prime}$ half of the $\alpha$-agglutinin (Ag $\left.\alpha\right)$ gene from $S$. cerevisiae was cloned into pMD18-T. The fragment DmAChE was amplified by PCR using primers AChE-F and AChEflag-R (all primers are listed in Table 1), adding a FLAG fragment. The amplified fragment was then re-inserted into pMD18-T. A 960-bp $\mathcal{N} o t \mathrm{I}-\mathrm{X} h \mathrm{I}$ fragment containing the Ag $\alpha$ gene was subsequently ligated into this construct. The target fragment was amplified by PCR using primers EntR-F and EntR-R and inserted into pENTR $^{\text {TM }}$ Directional TOPO vector (Invitrogen), which was subsequently recombined with pYes-DEST52 (Invitrogen). In order to replace the 114-bp secretion region of DmAChE with the 75-bp glucoamylase secretion peptide, a 1.4-kb fragment named GSU was amplified by PCR using primers pGSU-F1 and pGSUR. A 0.4-kb fragment named GSD2 was amplified by PCR using primers pGSD-F2 and pGSD-R with GSD1 (amplified by pGSDF1 and pGSD-R) as template. GSU and GSD2 was ligated by overlap PCR $(5 \mu \mathrm{L} 10 \times \mathrm{KOD}$ buffer, $4 \mu \mathrm{L} 2 \mathrm{mM}$ dNTPs, $2 \mu \mathrm{L}$ $25 \mathrm{mM} \mathrm{MgCl}_{2}, 1 \mu \mathrm{L}$ KOD plus, $2 \mu \mathrm{L}$ equal molar GSU and GSD2 as template, $48 \mu \mathrm{L}$ in total; $94^{\circ} \mathrm{C}, 3 \mathrm{~min} ; 94^{\circ} \mathrm{C}, 30 \mathrm{~s} ; 56^{\circ} \mathrm{C}$, $30 \mathrm{~s} ; 68^{\circ} \mathrm{C}, 90 \mathrm{~s}$ for 5 cycles followed by the addition of $1 \mu \mathrm{L}$

Table 1. Primers used in this study.

\begin{tabular}{|c|c|}
\hline $\begin{array}{l}\text { Primer } \\
\text { name }\end{array}$ & sequence $\left(5^{\prime}\right.$ to $\left.3^{\prime}\right)$ \\
\hline AChEF1 & CATATGATGGCCATCTCCTGTCGGCAGAG \\
\hline AChER1 & CTCGAGGAAAACCCTITTGGTTCGCAATGCGGC \\
\hline AChE-F & ATGGCCATCTCCTGTCGG \\
\hline \multirow[t]{2}{*}{ AChEflag-R } & GCGGCCGCCTTGTCATCGTCATCTTTATAATCACTTCCCGAA \\
\hline & TCGCCATC \\
\hline Aga- $\mathrm{F}$ & GCGGCCGCTAGCGCCAAAAGCTCTTITATCT \\
\hline Aga-R & CTCGAGCTAGAATAGCAGGTACGACAAAAGC \\
\hline EntR-F & CACCATGGCCATCTCCTGTCGG \\
\hline EntR-R & TAGAATAGCAGGTACGACAAAAGC \\
\hline pGSU-F1 & TGCGGGTGCATIITTTCAAGATAAAG \\
\hline \multirow[t]{2}{*}{ pGSU-R } & GAGAAAGAATGAAACTTTCAATGGCAAATTGAACAGTTGC \\
\hline & ATGGTGAAGGGGGCGGC \\
\hline \multirow[t]{2}{*}{ pGSD-F1 } & TTCTTCTCGTCCTCTCTTACTITTCTITGCTCGTTTCTGCTG \\
\hline & TCATCGATCGCCTGG \\
\hline \multirow[t]{2}{*}{ pGSD-F2 } & ATGCAACTGTTCAATTTGCCATTGAAAGTTTCATTCTITCTC \\
\hline & GTCCTCTCTTACTITTC \\
\hline pGSD-R & GACACGTTGGTGTTGGGGTTCC \\
\hline
\end{tabular}

$20 \mathrm{mM}$ pGSU-F1/pGSD-R followed by cycles). The resultant fragment was excised by $S n a B \mathrm{I}-B g / \mathrm{II}$ and ligated into pYesDEST52-AChE for the final construct. The whole construction process is shown in Figure 4.

\section{Yeast transformation}

The small scale transformation of $S$. cerevisiae was conducted using S.c.EasyComp. Transformation Kit (Invitrogen) as per the manufacturer's instructions.

\section{The induction of DmAChE expression}

The clones expressing pYES-DEST52-AChE were selected and cultured in medium supplemented with $2 \%$ glucose at $30^{\circ} \mathrm{C}$ and $300 \mathrm{rpm}$. The $\mathrm{OD}_{600}$ values were adjusted to 0.4 with fresh induction medium. Under the GAL1 promoter, the transcription of recombinant DmAChE was induced through the removal of glucose and the addition of galactose as the sole carbon source.

\section{Orthogonal test}

In the orthogonal test, YPD medium and SC minimal medium with $1 \%, 2 \%$ and $4 \%$ galactose were used. After specific periods of induction $(2,4,8,12$ and $24 \mathrm{~h}$ ), cultures were harvested and centrifuged at $1,500 \mathrm{rcf}$ for $5 \mathrm{~min}$ at $4^{\circ} \mathrm{C}$. The cells were resuspended and immediately used for activity and inhibition assays to determine growth rate comparisons and the effect of DmAChE expression and display.

\section{DmAChE activity assay}

DmAChE activity was assayed by the method reported previously [40] using $2 \mathrm{mM}$ final concentration of acetylthiocholine iodide as substrate and $2 \mathrm{mM}$ final concentration of DTNB as the chromogenic agent. The $S$. cerevisiae suspension $\left(\mathrm{OD}_{600}=1.0\right)$ served as the active enzyme. The $\mathrm{OD}_{412}$ values were determined once every $0.5 \mathrm{~min}$ for $15 \mathrm{~min}$ in $37^{\circ} \mathrm{C}$. The slope of the linear of absorbance versus time reflects the catalytic activity. The relative enzyme activity was acquired from the standard curve based on the results of commercial pure AChE enzyme. OP and CB insecticide stocks were prepared in acetone and diluted with phosphate buffered saline (PBS) buffer ( $\mathrm{pH}$ 7.6). For each sample, $10 \mu \mathrm{L}$ pesticide solution was pre-incubated with yeast suspension for $15 \mathrm{~min}$ at $37^{\circ} \mathrm{C}$. Uninduced $S$. cerevisiae (without expression of DmAChE on the surface) served as the negative control, whereas $S$. cerevisiae without pesticide incubation served as the positive control. The activity of the positive control was considered $100 \%$. The remaining activity of DmAChE is reflected as the slope of the following equation:

$$
\text { Inhibition } \operatorname{rate}(\%)=\left[\left(\Delta A_{0}-\Delta A_{t}\right) / \Delta A_{0}\right] \times 100
$$

$\Delta A_{0}:$ the change of absorbance of the positive control

$\Delta A_{t}:$ the change of absorbance of the pesticide incubated solution

When the inhibition rate $\geq 50 \%$, the pesticide was considered to be detected.

\section{Author Contributions}

Conceived and designed the experiments: JQL QB HW. Performed the experiments: JY SJW FFZ SCX JYL. Analyzed the data: JQL. Wrote the paper: JY QB JS WZ HW. 


\section{References}

1. Hofmann JN, Keifer MC, De Roos AJ, Fenske RA, Furlong CE, et al. (2010) Occupational determinants of serum cholinesterase inhibition among organophosphate-exposed agricultural pesticide handlers in Washington State. Occup Environ Med 67: 375-386.

2. Barrett JC, Vainio H, Peakall D, Goldstein BD (1997) 12th meeting of the Scientific Group on Methodologies for the Safety Evaluation of Chemicals: susceptibility to environmental hazards. Environ Health Perspect 105 Suppl 4: 699-737.

3. El Yazal J, Rao SN, Mehl A, Slikker W, Jr. (2001) Prediction of organophosphorus acetylcholinesterase inhibition using three-dimensional quantitative structure-activity relationship (3D-QSAR) methods. Toxicol Sci 63: 223-232.

4. Hofmann JN, Crowe J, Postma J, Ybarra V, Keifer MC (2009) Perceptions of environmental and occupational health hazards among agricultural workers in Washington State. AAOHN J 57: 359-371.

5. Hofmann JN, Checkoway H, Borges O, Servin F, Fenske RA, et al. (2010) Development of a computer-based survey instrument for organophosphate and $\mathrm{N}$-methyl-carbamate exposure assessment among agricultural pesticide handlers. Ann Occup Hyg 54: 640-650.

6. Whittaker VP (1990) The contribution of drugs and toxins to understanding of cholinergic function. Trends Pharmacol Sci 11: 8-13.

7. Berg L, Andersson CD, Artursson E, Hörnberg A, Tunemalm AK, et al. (2011) Targeting acetylcholinesterase: identification of chemical leads by high throughput screening, structure determination and molecular modeling. PLoS One 6: e26039.

8. Hicks DA, Makova NZ, Nalivaeva NN, Turner AJ (2013) Characterisation of acetylcholinesterase release from neuronal cells. Chem Biol Interact 203: 302308.

9. Coppage DL, Matthews E (1974) Short-term effects of organophosphate pesticides on cholinesterases of estuarine fishes and pink shrimp. Bull Environ Contam Toxicol 11: 483-488.

10. Rosenstock L, Keifer M, Daniell WE, McConnell R, Claypoole K (1991) Chronic central nervous system effects of acute organophosphate pesticide intoxication. The Pesticide Health Effects Study Group. Lancet 338: 223-227.

11. Savage EP, Keefe TJ, Mounce LM, Heaton RK, Lewis JA, et al. (1988) Chronic neurological sequelae of acute organophosphate pesticide poisoning. Arch Environ Health 43: 38-45.

12. Eskenazi B, Bradman A, Castorina R (1999) Exposures of children to organophosphate pesticides and their potential adverse health effects. Environ Health Perspect 107 Suppl 3: 409-419.

13. Blanc-Lapierre A, Bouvier G, Garrigou A, Canal-Raffin M, Raherison C, et al. (2012) [Chronic central nervous system effects of pesticides: state-of-the-art]. Rev Epidemiol Sante Publique 60: 389-400.

14. Steenland K, Jenkins B, Ames RG, O'Malley M, Chrislip D, et al. (1994) Chronic neurological sequelae to organophosphate pesticide poisoning. Am J Public Health 84: 731-736.

15. Albero B, Sanchez-Brunete C, Tadeo JL (2003) Determination of organophosphorus pesticides in fruit juices by matrix solid-phase dispersion and gas chromatography. J Agric Food Chem 51: 6915-6921.

16. Sandra P, Tienpont B, David F (2003) Multi-residue screening of pesticides in vegetables, fruits and baby food by stir bar sorptive extraction-thermal desorption-capillary gas chromatography-mass spectrometry. J Chromatogr A 1000: 299-309.

17. Fernandez M, Pico Y, Girotti S, Manes J (2001) Analysis of organophosphorus pesticides in honeybee by liquid chromatography-atmospheric pressure chemical ionization-mass spectrometry. J Agric Food Chem 49: 3540-3547.

18. Vinas P, Campillo N, Lopez-Garcia I, Aguinaga N, Hernandez-Cordoba M (2003) Capillary gas chromatography with atomic emission detection for pesticide analysis in soil samples. J Agric Food Chem 51: 3704-3708.

19. Villatte F, Marcel V, Estrada-Mondaca S, Fournier D (1998) Engineering sensitive acetylcholinesterase for detection of organophosphate and carbamate insecticides. Biosens Bioelectron 13: 157-164.

20. Hussein AS, Chacon MR, Smith AM, Tosado-Acevedo R, Selkirk ME (1999) Cloning, expression, and properties of a nonneuronal secreted acetylcholines- terase from the parasitic nematode Nippostrongylus brasiliensis. J Biol Chem 274: 9312-9319.

21. Mendelson I, Kronman C, Ariel N, Shafferman A, Velan B (1998) Bovine acetylcholinesterase: cloning, expression and characterization. Biochem J 334 Pt 1): 251-259.

22. Morel N, Massoulie J (1997) Expression and processing of vertebrate acetylcholinesterase in the yeast Pichia pastoris. Biochem J 328 (Pt 1): 121-129.

23. Simon S, Massoulie J (1997) Cloning and expression of acetylcholinesterase from Electrophorus. Splicing pattern of the $3^{\prime}$ exons in vivo and in transfected mammalian cells. J Biol Chem 272: 33045-33055.

24. Uccelletti D, De Jaco A, Farina F, Mancini P, Augusti-Tocco G, et al. (2002) Cell surface expression of a GPI-anchored form of mouse acetylcholinesterase in Klpmr1Delta cells of Kluyveromyces lactis. Biochem Biophys Res Commun 298: 559-565.

25. Mutero A, Fournier D (1992) Post-translational modifications of Drosophila acetylcholinesterase. In vitro mutagenesis and expression in Xenopus oocytes. J Biol Chem 267: 1695-1700.

26. Ueda M, Tanaka A (2000) Genetic immobilization of proteins on the yeast cell surface. Biotechnol Adv 18: 121-140.

27. Kondo A, Ueda M (2004) Yeast cell-surface display-applications of molecular display. Appl Microbiol Biotechnol 64: 28-40.

28. Gai SA, Wittrup KD (2007) Yeast surface display for protein engineering and characterization. Curr Opin Struct Biol 17: 467-473.

29. Gera N, Hussain M, Rao BM (2013) Protein selection using yeast surface display. Methods 60: 15-26.

30. Xu S, Wu A, Chen H, Xie Y, Xu Y, et al. (2007) Production of a novel recombinant Drosophila melanogaster acetylcholinesterase for detection of organophosphate and carbamate insecticide residues. Biomol Eng 24: 253-261.

31. Scholz NL, Truelove NK, Labenia JS, Baldwin DH, Collier TK (2006) Doseadditive inhibition of chinook salmon acetylcholinesterase activity by mixtures of organophosphate and carbamate insecticides. Environ Toxicol Chem 25: 12001207.

32. Tham LG, Perumal N, Syed MA, Shamaan NA, Shukor MY (2009) Assessment of Clarias batrachus as a source of acetylcholinesterase (AchE) for the detection of insecticides. J Environ Biol 30: 135-138.

33. Temeyer KB, Brake DK, Tuckow AP, Li AY, Perez de Leon AA (2013) Acetylcholinesterase of the sand fly, Phlebotomus papatasi (Scopoli): cDNA sequence, baculovirus expression, and biochemical properties. Parasit Vectors 6: 31.

34. Zhao P, Zhu KY, Jiang H (2010) Heterologous expression, purification, and biochemical characterization of a greenbug (Schizaphis graminum) acetylcholinesterase encoded by a paralogous gene (ace-1). J Biochem Mol Toxicol 24: 51-59.

35. Han Z, Chi G, Bai B, Liu G, Rao Q, et al. (2012) Chromogenic platform based on recombinant Drosophila melanogaster acetylcholinesterase for visible unidirectional assay of organophosphate and carbamate insecticide residues. Anal Chim Acta 720: 126-133.

36. Wu AB, Chen HD, Tang ZZ, Ye BW, Liu WJ, et al. (2008) Synthesis of Drosophila melanogaster acetylcholinesterase gene using yeast preferred codons and its expression in Pichia pastoris. Chem Biol Interact 175: 403-405.

37. Tan F, Wang L, Wang J, Wu X, Zhu H, et al. (2011) Enhanced pesticide sensitivity of novel housefly acetylcholinesterases: a new tool for the detection of residual pesticide contamination. Bioprocess Biosyst Eng 34: 305-314.

38. Boublik Y, Saint-Aguet P, Lougarre A, Arnaud M, Villatte F, et al. (2002) Acetylcholinesterase engineering for detection of insecticide residues. Protein Eng 15: 43-50.

39. Estrada-Mondaca S, Lougarre A, Fournier D (1998) Drosophila acetylcholinesterase: effect of post-translational [correction of post-traductional] modifications on the production in the baculovirus system and substrate metabolization. Arch Insect Biochem Physiol 38: 84-90.

40. Ellman GL, Courtney KD, Andres V Jr., Feather-Stone RM (1961) A new and rapid colorimetric determination of acetylcholinesterase activity. Biochem Pharmacol 7: 88-95. 